
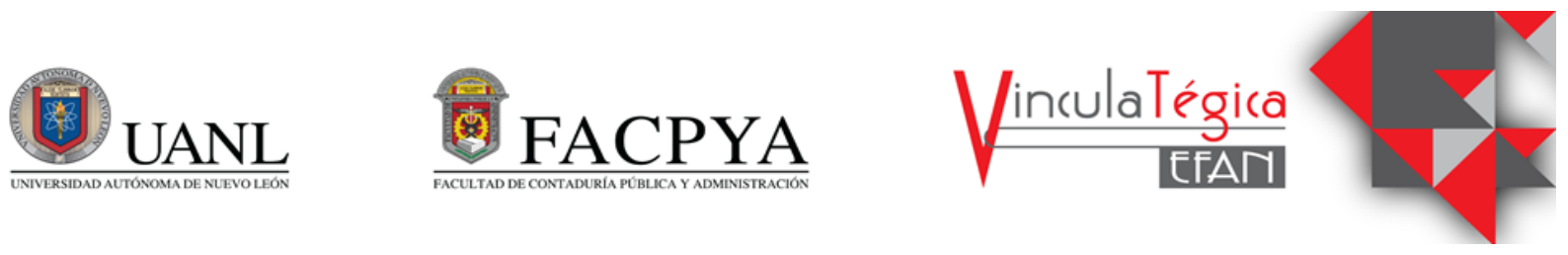

\title{
Satisfacción laboral: factores intrínsecos y extrínsecos del trabajador
}

\author{
Cristina del Pilar López Saldaña ${ }^{1}$, Maricela Carolina Peña Cárdenas ${ }^{2}$ y Adriana Guadalupe \\ Chávez Macías ${ }^{3}$ \\ ${ }^{1}$ Universidad Autónoma de Coahuila, Facultad de Contaduría y Administración Monclova, Coahuila, \\ México, cristinalopezsaldana@uadec.edu.mx, carretera 57 km.4.5, 8666390760 \\ ${ }^{2}$ Universidad Autónoma de Coahuila, Facultad de Contaduría y Administración Monclova, Coahuila, \\ México,maricela_pena_cardenas@uadec.edu.mx, carretera 57 km.4.5, 8666390760 \\ ${ }^{3}$ Universidad Autónoma de Coahuila, Facultad de Contaduría y Administración Monclova, Coahuila, \\ México,adrianachavez@uadec.edu.mx, carretera 57 km.4.5, 8666390760
}

\footnotetext{
Información del artículo revisado por pares

Fecha de aceptación: junio-2021

Fecha de publicación en línea: diciembre-2021

DOI: https://doi.org/10.29105/vtga7.1-170
}

\section{Resumen}

El objetivo de esta investigación consistió en estudiar la existencia de aspectos internos y externos de la satisfacción laboral en los trabajadores de pequeñas empresas. El planteamiento metodológico fue una investigación de campo tipo cuantitativa y descriptiva. Los resultados indicaron que existe un alto nivel de satisfacción laboral ya que los trabajadores manifestaron que: a) les agrada trabajar con sus compañeros, b) se sienten felices por los resultados logrados en su trabajo, c) se sienten bien haciendo su trabajo, d) consideran que la tarea que realiza es valiosa y e) la relación con sus superiores es cordial. Esto permitió concluir que el manejo de la organización en cuanto a factores internos como el desempeño del trabajo y el desarrollo personal o aspectos externos (la relación con los compañeros o el superior) representan un aspecto clave a considerar en el nivel de satisfacción laboral que manifiesten los trabajadores.

Palabras clave: satisfacción, laboral, factores, internos, externos.

\section{INTRODUCCIÓN}

Se considera que la satisfacción laboral es variable debido a que pueden aumentar o

\begin{abstract}
The objective of this research was to study the existence of both internal and external aspects of job satisfaction in workers in small companies. The methodological approach was a quantitative and descriptive field investigation. The results indicated that there is a high level of job satisfaction, since the workers stated that: a) they like working with their colleagues; b) they feel happy about the results achieved in their work; c) feel good doing their job; d) consider that the task they perform is valuable; e) the relationship with their superiors is cordial. This allowed to conclude that the management of the organization in terms of internal factors such as job performance and personal development; or external aspects such as the relationship with colleagues or with the superior, represent a key aspect to consider in the level of job satisfaction shown by workers.
\end{abstract}

Keywords: satisfaction, job, factors, internal, external.

disminuir las sensaciones agradables con base en las expectativas que tiene el trabajador y las necesidades que se vayan cubriendo. Una de 
las teorías más importantes sobre este tema es la que propuso Herzberg (1968) denominada teoría de los dos factores: uno de ellos son los factores de motivación que ayudan a aumentar la satisfacción del trabajador, los otros son los factores de higiene que si no existen o son inapropiados generan insatisfacción. La satisfacción laboral está basada en las diferencias percibidas por la persona entre lo que cree debe proporcionarle el empleo y lo que en realidad recibe como resultado o remuneración (Blum y Naylor, 1988), ya que es un resultado entre la aportación que lleva a cabo el empleado y lo que él espera en el ambiente de trabajo.

Chiavenato (2001) establece que los beneficios laborales representan medios esenciales para conservar la fuerza laboral dentro de un grado satisfactorio de moral y productividad. El análisis de la satisfacción y el desempeño laboral es importante para las empresas debido a que la postura de satisfacción en el trabajo está relacionada a una óptima culminación de los objetivos organizacionales (Chiavenato, 2007). Robbins (1998) menciona que el tener compañeros que ofrezcan camaradería y apoyo también aumenta la satisfacción laboral. Para Rico (2012) la satisfacción de un empleado con su empleo y su clima laboral es

\section{MARCO TEÓRICO}

Según Wright y Cropanzano (2000) la satisfacción laboral es comprendida como una respuesta emocional que emana al comparar la realidad laboral con las perspectivas vinculadas con esa realidad. Locke, citado por Paravic (2000), propone que la satisfacción laboral es el resultado de la diferencia entre lo que el empleado desea de su actividad laboral y lo que en realidad recibe moderada por el valor que para él representa, lo que deriva en que a menor diferencia entre lo que desea y lo que tiene se incrementará la satisfacción. Márquez (2001) comenta que la satisfacción podría conceptualizarse como el comportamiento del trabajador en cuanto a su propia actividad laboral, dicho comportamiento está cimentado en las convicciones y los valores que el trabajador aplica en su empleo y menciona también que un aspecto significativamente importante para los individuos que tienen a su cargo dirigir una organización.

Martínez (2007) expresa que la satisfacción laboral es considerada como un aspecto importante para los gerentes de las empresas por ser un elemento clave en el manejo del capital humano.

Esta investigación tiene como objetivo estudiar la existencia de diversos aspectos internos y externos de la satisfacción laboral en los trabajadores de pequeñas empresas de la ciudad de Monclova, Coahuila, con el propósito de ofrecer un diagnóstico de la situación prevaleciente en la organización. La satisfacción laboral simboliza un hecho que se manifiesta en el desempeño laboral, razón por la cual es necesario que las personas que tienen personal a su cargo asimilen lo que esto puede representar en los resultados de la organización.

La investigación está conformada de la siguiente manera: en primer lugar se presenta una inspección de la literatura sobre la satisfacción laboral, posteriormente en el segundo apartado se explica la metodología utilizada, en el tercer rubro se muestran los resultados conseguidos de la compilación de datos y por último se presentan las conclusiones del estudio.

la insatisfacción conduce a una disminución en la eficiencia organizacional.

Un concepto importante es el que presentan Davis y Newstrom (2007) quienes conceptualizan la satisfacción en el trabajo como un grupo de sensaciones positivas y negativas a través de las cuales los trabajadores visualizan su actividad laboral. Morillo (2006) conceptualiza la satisfacción laboral como el punto de vista positivo que tienen los empleados sobre su trabajo, manifestado por medio del grado de correlación existente entre las expectativas de los trabajadores con relación al trabajo, las compensaciones que éste le oferta, las relaciones interpersonales y la práctica gerencial. La satisfacción laboral puede ser distinguida por medio de ciertos componentes: que el trabajador sea adecuadamente 
remunerado acorde con lo que espera cada uno, que las condiciones del trabajo sean convenientes, lo cual repercute en un mejor desempeño, y que el empleado busque que su superior sea afable, accesible y le preste atención cuando se requiera (Smith et al., 1969; Rodríguez et al., 2010)

Uno de los elementos relevantes en cuanto a la satisfacción laboral son los beneficios laborales y/o remunerativos que según Palma (1999) representan el nivel de agrado en comparación con el incentivo financiero constante $\mathrm{o}$ adicional como remuneración por el trabajo que se desarrolla. Cavalcante (2004) menciona que si las retribuciones son convenientes se presentará un mayor nivel de satisfacción laboral debido a que los empleados visualizan una igualdad entre su actividad laboral y sus beneficios remunerativos.

El desarrollo personal es un componente para estudiar dentro de la satisfacción laboral ya que Palma (1999) lo define como la oportunidad que tiene el empleado de llevar a cabo actividades relevantes para su autorrealización. En lo referente a las variables asociadas con el desarrollo del trabajador se ha detectado que las mayores oportunidades de crecimiento profesional están de modo relevante relacionadas con altos niveles de satisfacción laboral, prominente compromiso con la institución y una pequeña intención de variar de empleo (Acker, 2004).

Otro elemento de la satisfacción laboral es el desempeño de tareas que según Palma (1999) representa la evaluación con la que vincula el empleado sus labores diarias en la organización en la que trabaja. Robbins y Judge (2013) expresan que hay una asociación entre la satisfacción laboral y factores asociados con el desempeño como la satisfacción al comprador para cargos de atención directa con el cliente o ausentismo y rotación. Lawler y Porter (1997) plantean que la vinculación satisfacción-desempeño puede ser opuesta: el grado de desempeño puede ser un factor causal en la satisfacción en el trabajo, siendo las compensaciones intrínsecas y extrínsecas variables de reforzamiento. La insatisfacción laboral es una señal de deterioro en la eficiencia organizacional que se asocia con una disminución en la productividad, ausentismo y otros comportamientos perjudiciales para los objetivos organizacionales (Paul, 2015).

Conforme a Palma (1999) las políticas administrativas son un factor a considerar dentro de la satisfacción laboral ya que representan el rango de conformidad ante las directrices o preceptos institucionales encaminados a regularizar la relación laboral, también menciona que las relaciones sociales muestran un aspecto a tomar en cuenta en la satisfacción laboral que define el nivel de agrado ante la interacción con otros miembros de la institución con quienes se colabora en las actividades diarias. Paravic (2000) cita a Locke quien confirma que es esencial que el trabajador conciba a sus compañeros como leales ya que si existe la ausencia de lealtad por parte de ellos es uno de los aspectos que genera mayor insatisfacción en el trabajo. Aritzeta y Ayestarán (2002) sostienen que la satisfacción laboral en las organizaciones será mayor en aquellas donde el trabajo se lleve a cabo en equipo que en las que se desarrolle en forma individual. Hegney et al. (2006) mencionan lo siguiente: la satisfacción laboral en el lugar de trabajo es suficientemente delimitado por la interrelación entre los trabajadores y las peculiaridades del entorno.

Un último aspecto que debe examinarse en la satisfacción laboral es la relación con la autoridad ya que Palma (1999) la explica como la opinión valorativa que lleva a cabo el empleado de su correlación con el superior inmediato y en relación a sus funciones cotidianas.

Newstom (2007) postula que los trabajadores insatisfechos caen en un abandono psicológico (se pierden mientras trabajan) en un abandono (ausentismo sin autorización, retiros temprano, prolongadas pausas o disminución del ritmo de trabajo) o comportamientos abiertos de ataque y venganza por presuntos errores.

Johns (1996) identificó al

reconocimiento, los beneficios, las condiciones de trabajo, la supervisión, los compañeros de trabajo y las políticas de la empresa como los criterios de medición de la 
satisfacción laboral. Los resultados del trabajo de la investigación de Rodríguez et al. (2010) muestran que las remuneraciones económicas y las condiciones físicas de la institución son los factores condicionantes de la satisfacción laboral. Chian y San Martín (2015) en su estudio encontraron correlaciones estadísticamente relevantes entre las variables de desempeño laboral y satisfacción laboral, además resaltan las correlaciones positivas entre los niveles de desempeño y satisfacción en la correlación con el superior inmediato para las mujeres y con la productividad para las personas de sexo femenino. Los resultados de Ramos y Padilla (2016) demuestran que en el entorno laboral de la institución que estudiaron hay mayor nivel de satisfacción en los aspectos intrínsecos y que los mejores calificados fueron: el logro personal y responsabilidad-realización ya que los trabajadores manifestaron su deseo por lograr metas de crecimiento personal.

\section{MÉTODO}

El planteamiento metodológico perteneció a una investigación de campo tipo cuantitativa y descriptiva. Los datos se recabaron a través de una encuesta aplicada a 313 trabajadores de doce pequeñas empresas en la ciudad de Monclova, Coahuila, al tratarse de una muestra no probabilística la que se examinó para realizar este estudio. Se utilizó el instrumento de medición titulado Escala de Satisfacción Laboral SL-SPC diseñado por Palma (1999) que comunica como válido y confiable con un Alfa de Cronbach de 0.796.

\section{RESULTADOS}

En primer lugar se presentan las características demográficas de las personas encuestadas y después la posición de las empresas con respecto a las variables analizadas. Una vez que se trataron los datos por medio del software estadístico SPSS se obtuvieron los
Pozo et al. (2005) finalizan: las variables vinculadas a las relaciones interpersonales (con superiores o compañeros) han generado una extensa literatura donde se ha demostrado su rol en el pronóstico de la satisfacción laboral, del bienestar general de los trabajadores, de los resultados institucionales y la ejecución individual de los empleados. Vargas (2012) concluye que entre los componentes básicos de la motivación y satisfacción laboral se encuentran las relaciones y la comunicación informal, así como la figura o impresión del proceder del empresario o director general. Buckingham y Coffman (2003) establecen que la relación de la autoactualización gerencial y la satisfacción laboral de los trabajadores se visualiza en procesos humanos de las relaciones interpersonales: la comunicación efectiva, una adecuada toma de decisiones, un ambiente participativo, respeto esencial a las personas y aprendizaje mutuo.

Para la evaluación de los 30 reactivos se aplicó una escala de tipo Likert de 1 a 5 (Totalmente en desacuerdo, En desacuerdo, Indeciso, De acuerdo y Totalmente de acuerdo). Para realizar la medición de las variables en estudio: remuneraciones, políticas administrativas, relaciones sociales, desarrollo personal, desempeño de tareas y relación con la autoridad, así como para detectar las relaciones investigadas utilizando la técnica del coeficiente Rho de Spearman. Los datos que se recabaron fueron procesados a través del paquete estadístico informático SPSS.

resultados referentes a las características que se exhiben en la Tabla 1 representando mayoría los siguientes aspectos: el 54.3\% son del sexo femenino, el $45.4 \%$ cuentan con estudio de preparatoria, el $38.3 \%$ tienen una antigüedad laboral en la empresa de menos de un año y por último el $38.6 \%$ realizan cargos de atención a clientes. 
Tabla 1. Características demográficas.

$\begin{array}{lrr}\text { Sexo del encuestado } & 143 & \\ \text {-Hombre } & 170 & 45.7 \\ \text {-Mujer } & & 54.3 \\ \text { Escolaridad del encuestado } & 94 & 30.0 \\ \text {-Secundaria } & 142 & 45.4 \\ \text {-Preparatoria } & 77 & 24.6 \\ \text {-Profesional } & 120 & \\ \text { Antigüedad laboral del encuestado } & 87 & 38.3 \\ \text { < de 1 año } & 28 & 27.8 \\ \text { 1 año a < de 2 años } & 78 & 9.0 \\ \text { 2 años a < de 3 años } & & 24.9 \\ \text { 3 años o más } & 78 & \\ \text { Edad del encuestado } & 72 & 24.9 \\ \text { 18-20 } & 62 & 23.0 \\ \text { 21-25 } & 29 & 19.8 \\ \text { 26-30 } & 72 & 9.3 \\ \text { 31-35 } & & 23.0 \\ \text { >de 35 años } & 30 & \\ \text { Puesto del encuestado } & 50 & 9.6 \\ \text {-Supervisión } & 121 & 16.0 \\ \text {-Administrativo } & 112 & 38.6 \\ \text {-Atención a clientes } & & 35.8 \\ \text {-Servicios generales } & \end{array}$

Fuente: Elaboración propia.

En la Tabla 2 se presentan las puntuaciones promedio de cada una de las seis variables que se analizaron donde se puede identificar que la variable que resultó con un mayor puntaje es la de Desarrollo Personal (3.90) y la de puntuación más baja con respecto a la media es Políticas Administrativas (2.61). Al obtener estos resultados se estableció que el desarrollo personal es predominante para los trabajadores en cuanto a su satisfacción laboral ya que ellos están contentos y disfrutan las actividades que desempeñan, además de que se sienten realizados profesionalmente.

Tabla 2. Puntuaciones promedio por variable.

$\begin{array}{cc}\text { Variable } & \text { Media } \\ \text { Remuneraciones } & 3.10 \\ \text { Políticas administrativas } & 2.61 \\ \text { Relaciones sociales } & 3.57 \\ \text { Desarrollo personal } & 3.90 \\ \text { Desempeño de tareas } & 3.42 \\ \text { Relación con la autoridad } & 3.62\end{array}$

Fuente: Elaboración propia.

Con la finalidad de observar el nivel de satisfacción prevaleciente en la Tabla 3 se muestra la frecuencia de respuestas por item conjuntadas por variable donde se puede percibir que en cuatro de las variables estudiadas los puntos de medición presentaron una puntuación mayor al 55\% en lo referente a un alto nivel de satisfacción. En cuanto a las políticas administrativas se puede observar que la mayoría reflejó un alto grado de satisfacción. Por su parte las remuneraciones en todas las preguntas se obtuvo una menor puntuación en cuanto al nivel de satisfacción alto.

Los puntos que sobresalen porque alcanzaron un porcentaje mayor al $79 \%$ en 
cuanto a la existencia de satisfacción laboral en los empleados son los siguientes: que les agrada trabajar con sus compañeros, que se sienten felices por los resultados que logran en su trabajo, que haciendo su trabajo se sienten bien consigo mismo, que la tarea que realizan es tan valiosa como cualquier otra y que la relación que tienen con sus superiores es cordial.

Tabla 3. Nivel de satisfacción: frecuencia de respuestas conjuntadas por variable $(n=313)$.

\begin{tabular}{|c|c|c|c|}
\hline & Baja & Regular & Alta \\
\hline \multicolumn{4}{|l|}{ Remuneraciones } \\
\hline Mi sueldo esta correcto con relación en la labor que realizo & $119 / 38.1 \%$ & $91 / 29.1 \%$ & $103 / 32.8 \%$ \\
\hline Me siento bien con lo que gano & $83 / 26.6 \%$ & $98 / 31.3 \%$ & $132 / 42.1 \%$ \\
\hline Percibo que el sueldo que me pagan es bastante aceptable & $83 / 26.5 \%$ & $94 / 30.0 \%$ & $136 / 43.5 \%$ \\
\hline Mi empleo me permite cubrir mis expectativas económicas & $74 / 23.6 \%$ & $83 / 26.5 \%$ & $156 / 49.8 \%$ \\
\hline \multicolumn{4}{|l|}{ Políticas administrativas } \\
\hline Siento que doy menos de lo que recibo de la institución & $109 / 34.8 \%$ & $86 / 27.5 \%$ & $118 / 37.7 \%$ \\
\hline La sensación que tengo de mi trabajo es que me están respetando & $51 / 16.3 \%$ & $74 / 23.6 \%$ & $188 / 60.1 \%$ \\
\hline Me gusta mi horario & $86 / 27.5 \%$ & $68 / 21.7 \%$ & $159 / 50.8 \%$ \\
\hline El horario de trabajo me resulta cómodo & $72 / 23.0 \%$ & $68 / 21.7 \%$ & $173 / 55.3 \%$ \\
\hline El esfuerzo de laborar de horas extras me es reconocido & $97 / 31.0 \%$ & $82 / 26.2 \%$ & $134 / 42.8 \%$ \\
\hline \multicolumn{4}{|l|}{ Relaciones sociales } \\
\hline El ambiente establecido por mis compañeros es perfecto & $28 / 9.0 \%$ & $60 / 19.2 \%$ & $225 / 71.8 \%$ \\
\hline Me agrada trabajar con mis compañeros & $24 / 7.7 \%$ & $30 / 9.6 \%$ & $259 / 82.7 \%$ \\
\hline Prefiero tener cercanía con las personas con las que trabajo & $65 / 20.8 \%$ & $63 / 20.1 \%$ & $185 / 59.1 \%$ \\
\hline La solidaridad es una característica de nuestro grupo de trabajo & $35 / 11.2 \%$ & $58 / 18.5 \%$ & $220 / 70.3 \%$ \\
\hline \multicolumn{4}{|l|}{ Desarrollo personal } \\
\hline Aprecio que el trabajo que realizo es justo para mi forma de ser & $41 / 13.1 \%$ & $61 / 19.5 \%$ & $211 / 67.4 \%$ \\
\hline Mi trabajo me permite desarrollarme personalmente & $41 / 13.1 \%$ & $57 / 18.2 \%$ & $215 / 68.7 \%$ \\
\hline Disfruto cada labor que realizo en mi trabajo & $38 / 12.2 \%$ & $59 / 18.8 \%$ & $216 / 69.0 \%$ \\
\hline Me siento feliz por los resultados que logro en mi trabajo & $22 / 7.0 \%$ & $41 / 13.1 \%$ & $250 / 79.9 \%$ \\
\hline Mi trabajo me hace sentir realizado profesionalmente & $56 / 17.9 \%$ & $83 / 26,5 \%$ & $174 / 55.6 \%$ \\
\hline Haciendo mi trabajo me siento bien conmigo & $20 / 6.4 \%$ & $44 / 14.1 \%$ & $249 / 79.5 \%$ \\
\hline \multicolumn{4}{|l|}{ Desempeño de tareas } \\
\hline El trabajo que desempeño es tan valioso como cualquier otro & $19 / 6.1 \%$ & $33 / 10.5 \%$ & $261 / 83.4 \%$ \\
\hline Me siento realmente útil con la labor que realizo & $25 / 8.0 \%$ & $42 / 13.4 \%$ & $246 / 78.6 \%$ \\
\hline Las tareas que realizo las percibo como importantes & $82 / 26.2 \%$ & $55 / 17.6 \%$ & $176 / 56.3 \%$ \\
\hline Mi trabajo lo disfruto & $52 / 16.6 \%$ & $64 / 20.4 \%$ & $197 / 63.0 \%$ \\
\hline Me gusta el trabajo que realizo & $28 / 8.9 \%$ & $50 / 16.0 \%$ & $235 / 75.1 \%$ \\
\hline \multicolumn{4}{|l|}{ Relación con la autoridad } \\
\hline Mi jefe(a) es tolerante & $35 / 11,2 \%$ & $66 / 21.1 \%$ & $212 / 67.7 \%$ \\
\hline Es agradable la actitud de mi jefe(a) al preguntar sobre mi trabajo & $32 / 10.2 \%$ & $65 / 20.8 \%$ & $216 / 69.0 \%$ \\
\hline Llevarme bien con mi jefe(a) beneficia la calidad de mi trabajo & $43 / 13.7 \%$ & $50 / 16.0 \%$ & $220 / 70.3 \%$ \\
\hline La comunicación que tengo con mis superiores es cordial & $25 / 8.0 \%$ & $40 / 12.7 \%$ & $248 / 79.3 \%$ \\
\hline Me siento cómodo con mi jefe(a) & $70 / 22.3 \%$ & $44 / 14.1 \%$ & $199 / 63.6 \%$ \\
\hline El jefe(a) aprecia el esfuerzo que realizo en mi trabajo & $38 / 12.1 \%$ & $64 / 20.4 \%$ & $211 / 67.5 \%$ \\
\hline
\end{tabular}

Fuente: Elaboración propia.

Tomando como base la respuesta de los trabajadores de las empresas estudiadas los resultados por variable que se muestran en la
Tabla 4 reflejan lo siguiente: en cuatro de ellas los empleados tienen una alta satisfacción laboral.

Tabla 4. Nivel de satisfacción por variable.

$\begin{array}{cccc} & \text { Bajo } & \text { Regular } & \text { Alto } \\ \text { Remuneraciones } & 90 / 28.7 \% & 91 / 29.2 \% & 131 / 42 \% \\ \text { Políticas administrativas } & 83 / 26.5 \% & 76 / 24.3 \% & 154 / 49.2 \% \\ \text { Relaciones sociales } & 38 / 12.2 \% & 53 / 16.9 \% & 222 / 70.9 \% \\ \text { Desarrollo personal } & 36 / 11.5 \% & 58 / 18.5 \% & 219 / 70.0 \% \\ \text { Desempeño de tareas } & 41 / 13.1 \% & 49 / 15.7 \% & 223 / 71.2 \% \\ \text { Relación con la autoridad } & 40 / 12.8 \% & 55 / 17.6 \% & 218 / 69.6 \%\end{array}$

Fuente: Elaboración propia. 
En las Tablas 5 y 6 se exhiben la frecuencia de respuestas por variable y cada una de las características demográficas donde sobresalen los siguientes puntos: en todas las variables las mujeres, las personas que tienen más de 35 años de edad y los que desempeñan puestos de supervisión manifestaron un mayor porcentaje en lo referente a una alta satisfacción laboral, mientras que los trabajadores que cuentan con estudios profesionales y los que tienen una antigüedad laboral de dos a menos de tres años presentaron en cinco de las seis variables estudiadas una mayor satisfacción laboral.
Los resultados arrojaron diferencias significativas en cuanto a una alta satisfacción laboral en las variables siguientes: en remuneraciones los que tienen menos de un año laborando un (38.9\%) y los de dos años a menos de tres un $(51.8 \%)$, en relaciones sociales los que tiene de 18 a 20 años de edad un $(6.1 \%)$ y los mayores de 35 años un $(80.2 \%)$, en desarrollo personal los que desempeñan el puesto de atención a clientes un $(62.7 \%)$ y lo de supervisión un $(90.6 \%)$ y en políticas administrativas los que tienen un puesto de servicios generales un $(43.9 \%)$ y los de supervisión un $(71.3 \%)$.

Tabla 5. Frecuencia de respuestas por variable y característica demográfica.

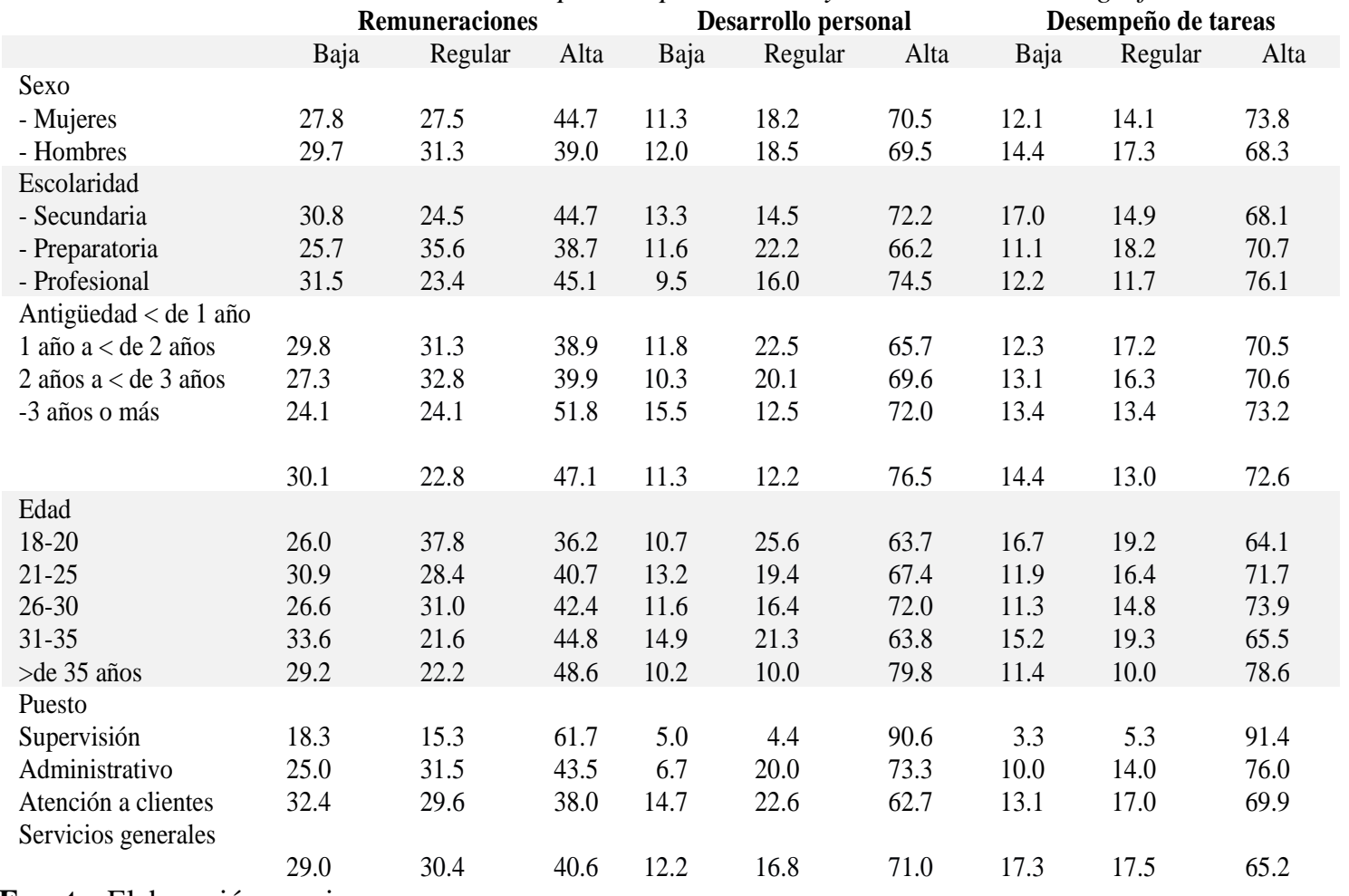

Fuente: Elaboración propia.

Tabla 6. Frecuencia de respuestas por variable y característica demográfica.

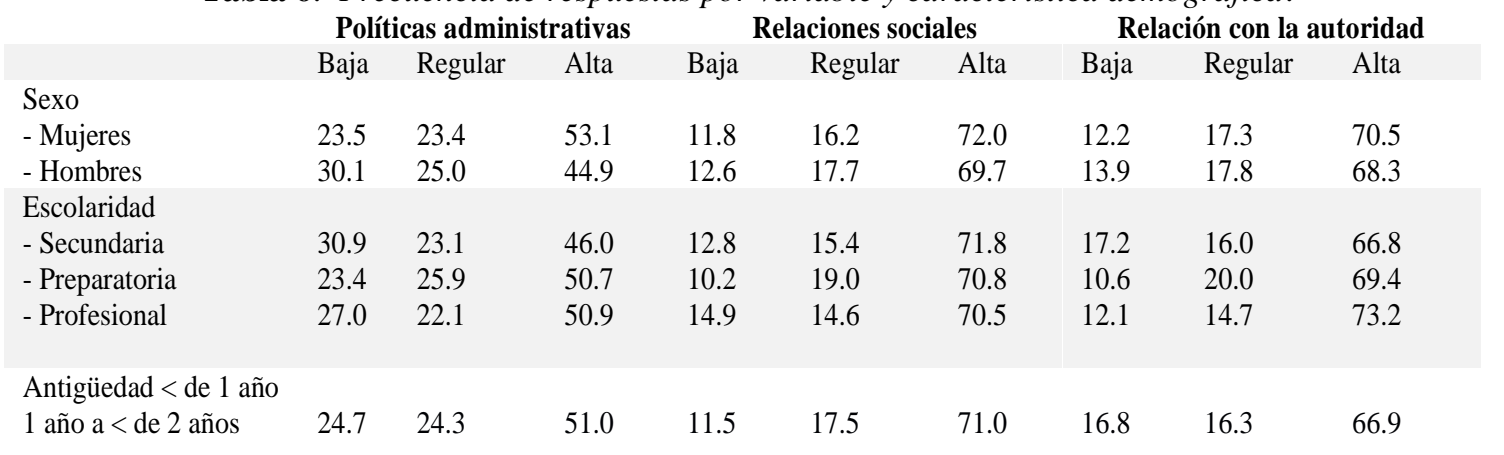




$\begin{array}{lrrrrrrrrr}2 \text { años a < de 3 años } & 28.5 & 27.1 & 44.4 & 12.6 & 16.7 & 70.7 & 9.8 & 17.0 & 73.2 \\ \text { 3 años o más } & 15.7 & 25.0 & 59.3 & 7.1 & 15.2 & 77.7 & 10.1 & 15.5 & 74.4 \\ & & & & & & & & & \\ & 31.0 & 20.3 & 48.7 & 14.4 & 16.7 & 68.9 & 11.5 & 20.7 & 67.8 \\ \text { Edad } & & & & & & & & & \\ 18-20 & 29.5 & 30.5 & 40.0 & 16.7 & 21.2 & 62.1 & 15.4 & 18.2 & 66.4 \\ 21-25 & 21.4 & 26.4 & 52.2 & 10.0 & 18.0 & 72.0 & 13.0 & 19.2 & 67.8 \\ 26-30 & 26.5 & 24.2 & 49.3 & 10.5 & 15.7 & 73.8 & 10.5 & 21.0 & 68.5 \\ \text { 31-35 } & 28.3 & 18.0 & 53.7 & 14.7 & 19.8 & 65.5 & 14.4 & 17.8 & 67.8 \\ \text { >de 35 años } & 27.8 & 17.5 & 54.7 & 9.4 & 10.4 & 80.2 & 11.8 & 12.0 & 76.2 \\ \text { Puesto } & & & & & & & & & \\ \text { - Supervisión } & 14.7 & 14.0 & 71.3 & 3.4 & 13.3 & 83.3 & 9.5 & 7.2 & 83.3 \\ \text { - Administrativo } & 22.0 & 19.2 & 58.8 & 9.0 & 14.5 & 76.5 & 8.0 & 15.3 & 76.7 \\ \text { - Atención a clientes } & 27.8 & 27.2 & 45.0 & 14.4 & 17.6 & 68.0 & 13.2 & 22.3 & 64.5 \\ \text { - Servicios generales } & & & & & & & & & \\ & 30.4 & 25.7 & 43.9 & 13.4 & 18.0 & 68.6 & 15.8 & 16.0 & 68.2\end{array}$

Nota: Resultados divididos en dos partes.

Fuente: Elaboración propia.

En la tabla 7 se presentan los resultados que se obtuvieron en cuanto a la relación de las variables, empleando la correlación de Rho de Spearman y también se muestra el nivel de significancia. Los datos una vez que se procesaron mostraron asociaciones

significativas positivas $(\mathrm{p}<0.01)$ además de una correlación positiva en todas las variables estudiadas. Las que presentaron una relación más fuerte $(\mathrm{r}=0.842)$ son la de Desarrollo Personal con Relaciones Sociales.

Tabla 7. Relación y significancia entre las variables analizadas.

\begin{tabular}{|c|c|c|c|c|c|c|}
\hline & Remuneraciones & $\begin{array}{c}\text { Políticas } \\
\text { administrativas }\end{array}$ & $\begin{array}{c}\text { Relaciones } \\
\text { sociales }\end{array}$ & $\begin{array}{c}\text { Desarrollo } \\
\text { personal }\end{array}$ & $\begin{array}{c}\text { Desempeño } \\
\text { de tareas }\end{array}$ & $\begin{array}{l}\text { Relación con } \\
\text { la autoridad }\end{array}$ \\
\hline Remuneraciones & 1 & & & & & \\
\hline Políticas administrativas & $\begin{array}{l}.787 \\
.000\end{array}$ & 1 & & & & \\
\hline Relaciones sociales & $\begin{array}{l}.703 \\
.000\end{array}$ & $\begin{array}{l}.760 \\
.000\end{array}$ & 1 & & & \\
\hline Desarrollo personal & $\begin{array}{l}.711 \\
.640\end{array}$ & $\begin{array}{l}.795 \\
.000\end{array}$ & $\begin{array}{l}.842 \\
.000\end{array}$ & 1 & & \\
\hline Desempeño de tareas & $\begin{array}{l}.763 \\
.000\end{array}$ & $\begin{array}{l}.807 \\
.000\end{array}$ & $\begin{array}{l}.756 \\
.000\end{array}$ & $\begin{array}{l}.774 \\
.000\end{array}$ & 1 & \\
\hline Relación con la autoridad & $\begin{array}{l}.751 \\
.000\end{array}$ & $\begin{array}{l}.775 \\
.000\end{array}$ & $\begin{array}{l}.810 \\
.000\end{array}$ & $\begin{array}{l}.830 \\
.000\end{array}$ & $\begin{array}{l}.749 \\
.000\end{array}$ & 1 \\
\hline
\end{tabular}

Fuente: Elaboración propia.

\section{CONCLUSIONES}

Esta investigación tuvo como objetivo estudiar la existencia de diversos aspectos internos y externos de la satisfacción laboral en los trabajadores de pequeñas empresas de la ciudad de Monclova, Coahuila, con el propósito de ofrecer un diagnóstico de la situación prevaleciente en la organización.

Los resultados que se lograron sobre las empresas estudiadas indican lo siguiente: que en cuatro de las seis variables analizadas se obtuvo un porcentaje elevado en cuanto a un alto nivel de satisfacción, los trabajadores manifestaron que les agrada trabajar con sus compañeros, otro aspecto a resaltar es que se sienten felices por los resultados que logran en su trabajo, que se sienten bien haciendo su trabajo, que consideran que la tarea que realizan es tan valiosa como cualquier otra y que la relación que tienen con sus superiores es cordial. Dichos resultados tienen una gran semejanza con los trabajos de (Pozo et al. 2005; Chian y San Martín, 2015; de Ramos y Padilla, 2016) debido a que encontraron una relación relevante de estos mismos aspectos con un alto nivel de satisfacción laboral.

En cuanto a las respuestas alcanzadas contemplando las características demográficas sobresalen los siguientes puntos: los 
trabajadores del género femenino, los empleados que tienen una edad mayor a los 35 años y los supervisores manifiestan un alto nivel de satisfacción laboral.

También los resultados manifiestan relaciones importantes afirmativas $(\mathrm{p}<0.01)$, además una correlación positiva en cada una de las variables analizadas y las variables que presentaron una asociación más significativa $(r=0.842)$ fueron la de desarrollo personal con relaciones sociales.

Considerando el informe logrado la conclusión es: el manejo por parte de la organización en cuanto a factores internos como el desempeño del trabajo y el desarrollo personal o aspectos externos como la relación con los compañeros o con el superior representan un aspecto clave a considerar en el nivel de satisfacción laboral que manifiestan los trabajadores.

El llevar a cabo la realización de este trabajo de investigación resulta pertinente para que las personas que tienen empleados a su cargo reconozcan que estos factores representan un papel importante en el funcionamiento de una empresa. 


\section{REFERENCIAS}

Acker, J. (2004). The effect of organizational conditions (role conflict, role ambiguity, opportunities for professional development, and social support) on job satisfaction and intention to leave among social workers in mental health care. Community Mental Health Journal, 40(1), 6573.

Aritzeta, A. y Ayestarán, S. (2002). Utilidad de los equipos de trabajo para incrementar la participación, autogestión, interdependencia, satisfacción e integración de conductas cooperativas y competitivas. Ciriec, Revista de economía pública, social y cooperativa, 40, 195-212.

Blum, M. y Naylor, J. (1988). Psicología industrial. Sus fundamentos teóricos y sociales. México: Trillas.

Buckingham, M. y Curt, C. (2003). Primero Rompa Todas las Reglas. México, Norma.

Cavalcante, J. (2004). Satisfacción en el trabajo de los directores de escuelas secundarias públicas de la región de Jacobina (Bahía-Brasil). España: Universidad Autónoma de Barcelona. http://hdl.handle.net/10803/

Chiang, V. M. M. y San Martín, N. N. J. (2015). Análisis de la satisfacción y el desempeño laboral en los funcionarios de la Municipalidad de Talcahuano. Ciencia \& Trabajo, 17(54).

Chiavenato, I. (2001). Administración de Recursos Humanos. Santa Fe de Bogotá: McGraw-Hill Interamericana.

Chiavenato, I. (2007). Introducción a la Teoría General de la Administración. México: McGraw-Hill Interamericana Editores S.A.

Davis, K. y Newstrom, W. (2007). El comportamiento humano en el trabajo. México: McGraw-Hill. Herzberg, F. I. (1968). One More Time, How Do You Motivate Employees.

Hegney, D., Plank, A. y Parker, V. (2006). Extrinsic and intrinsic work values: Their impact on job satisfaction in nursing. Journal of Nursing Management, 14, 271-281.

Johns, G. (1996). Organizational behavior: understanding and managing life at work. New York: Harper Collins College Publishers.

Lawler, E. y Porter, L. (1997). The effect of performance on job satisfaction. Industrial Relations, 7(20), 20-28.

Martínez, C. N. (2007). El caso de la Satisfacción Laboral. Recursos Humanos y Management Empresarial. Cuadernos de CC.EE. y EE., 52, 75-101.

Márquez, $\quad$ P. $\quad$ M. (2001). Satisfacción laboral. http://www.gestiopolis.com/recursos/documentos/fulldocs/rrhh/satlab.htm

Morillo, I. (2006). Nivel de satisfacción del personal académico del Instituto Pedagógico de Miranda José Manuel Sisso Martínez en relación con el estilo de liderazgo del jefe del departamento. Sapiens, 7(1), 43-57.

Newstrom, J. W. (2007). Comportamiento humano en el trabajo. México: McGraw Hill.

Palma, S. (1999). Elaboración y validación de una Escala de Satisfacción Laboral SL-SPC para trabajadores de Lima metropolitana. Revista teoría e investigación en Psicología, 9(1), $27-$ 34.

Paravic, T. (2000). Satisfacción laboral de mujeres académicas de una universidad en Chile. Ciencia y Enfermería. Revista Interamericana de Investigación, (6),1-5.

Paul, A. (2015). Job satisfaction: its determinants and relationship with employee performance in the tanzanian banking sector. [Tesis de maestría]. Universidad Abierta de Tanzania.

Pozo, M., C. A., Morillejo, E., Hernández P., S. y Martos M., M. J. (2005). Determinantes de la satisfacción laboral en trabajadores de la administración pública: el valor de las relaciones interpersonales en el lugar de trabajo. Ansiedad y Estrés, 11(2-3), 247-264.

Ramos, R. G. y Padilla, B. L. E. (2016). Satisfacción laboral del personal administrativo de la administración central de la universidad autónoma de Zacatecas. Red Internacional de Investigadores en Competitividad. 
Rico, B. P. (2012). Satisfacción laboral de los asalariados en España. Revista de Métodos Cuantitativos para la Economía y la Empresa, 137-158.

Robbins, S. (1998). Fundamentos de comportamiento organizacional. Pearson Educación.

Robbins, S. y Judge, T. (2013). Comportamiento organizacional. México: Pearson Educación de México.

Rodríguez, D., Núñez, L. y Cáceres, A. (2010). Estudio comparativo de la satisfacción laboral universitaria en el núcleo de Anzoátegui de la Universidad de Oriente. Investigación y Posgrado, 63-68.

Smith, P. C., Kendall, L.M. y Hulin, C. L. (1969). The measurement of satisfaction in work and retirement. Chicago: Rand McNally.

Vargas, T. J. A. (2012). Organización del trabajo y satisfacción laboral: un estudio de caso en la industria del calzado. Nova Scientia, 4(7).

Wright, T. A. y Cropanzano, R. (2000). Psychological well-being and job satisfaction as predictors of job performance. Journal of Occupational Health Psychology, 5(1), 84-94. 